\title{
Translating Chicana Rap: Snow Tha Product
}

\author{
Adriana Onita \\ University of Alberta, Edmonton
}

This project examines rap lyrics, interviews, and music videos by Chicana artist Snow Tha Product to show how rap has been culturally translated, performed, and appropriated by females in order to "flip the script," or subvert the dichotomous model of female sexuality that has been imposed upon them. Weaving insights from three academic fields (cultural translation, Chican@ studies, and hip-hop feminism), this paper also aims to creatively expand the definition of translation by positioning rap music as a performative language in its own right, capable of encoding and translating complex cultural issues related to race, gender, and sexuality. 